\title{
Metabolic influences that regulate dendritic cell function
}

\section{in tumors}

\author{
Han Dong ${ }^{1}$ and Timothy N. J. Bullock ${ }^{2 *}$ \\ 1 Experimental Pathology Program, University of Virginia, Charlottesville, VA, USA \\ ${ }^{2}$ Department of Pathology, University of Virginia, Charlottesville, VA, USA
}

\section{Edited by:}

Kristian Michael Hargadon,

Hampden-Sydney College, USA

Reviewed by:

Pierre Sonveaux, University of Louvain (UCL) Medical School,

Belgium

Thomas Weichhart, Medical

University of Vienna, Austria

*Correspondence:

Timothy N. J. Bullock, Department of Pathology, University of Virginia,

Room G526, MR6 Building, 345

Crispell Drive, Charlottesville, VA

22908, USA

e-mail: tb5v@virginia.edu
Dendritic cells $(D C)$ are critical regulators of both activation and tolerance in the adaptive immune response. The dual nature of DC immunoregulatory function depends on their differentiation and activation status. DC found within the tumor microenvironment (TME) and tumor-draining lymph node often exist in an inactive state, which is thought to limit the adaptive immune response elicited by the growing tumor. The major determinants of DC activation and the functional alterations in DC that result from integrating exogenous stimuli have been well investigated. Extensive efforts have been made to elucidate how the TME contributes to the inactivated/dysfunctional phenotype of tumor-associated DC (TADC). Although performed predominantly on in vitro DC cultures, recent evidence indicates that DC undergo required, coordinated alterations in their metabolism upon activation, and dysregulated metabolism in TADC is associated with their reduced immunostimulatory capacity. In this review, we will focus on the role of glycolysis and fatty acid metabolism in DC activation and function and discuss how these metabolic pathways may be regulated in TADC. Further, we consider the need for developing novel experimental approaches to assess metabolic choices in vivo, and the necessity for integrating metabolic regulation into the optimized development of DC for tumor vaccines and immunotherapy for cancer.

Keywords: dendritic cell, tumor-associated dendritic cell, activation, metabolism, glycolysis, oxidative phosphorylation, lipid metabolism, cancer immunotherapy

\section{INTRODUCTION}

Dendritic cells (DC) serve as sentinels of the immune system. They constantly acquire antigen $(\mathrm{Ag})$ from their environment and degrade it into short peptides that are presented at the cell surface in association with MHC molecules for surveillance by $\mathrm{T}$ cells. The inflammatory context in which DC exist influences their expression of critical co-stimulatory molecules and cytokines (Figure 1B) that provide the context for Ag presentation. Factors that promote the expression of co-stimulatory molecules and cytokines support the activation, expansion, and survival of responding $\mathrm{T}$ cells. In the absence of co-stimulation, DC present $\mathrm{Ag}$ in a manner that induces tolerance in the specific $\mathrm{T}$ cell repertoire, by mechanisms such as deletion (1) and anergy (2). During infection, inflammatory cytokines such as TNF $\alpha$ and type1 interferons (IFN-1), or pathogen associated molecular patterns (PAMPs), induce a program of activation that initiates the CCR7dependent migration of DC from the periphery to draining lymph nodes $(3,4)$. Additional stimulation via CD40 can further raise the activation state of DC, in part by inducing the expression of CD70 (Figure 1B) (5-7), leading to what is referred to as a licensed T cell response. While these basic tenets of DC activation have been well investigated, and extensively reviewed elsewhere $(8,9)$, recent studies have brought to light metabolic transitions in DC that are necessary for them to attain full function, or can regulate their functional activation. Here, we discuss the impact of these metabolic alterations on DC function; how metabolic pathways may be regulated in tumor-associated DC (TADC); and given the immature state of
DC often found in tumors [and the negative prognosis associated with such immaturity $(10,11)]$ we consider the influence of the tumor microenvironment (TME) on these functions.

\section{REOUIREMENT FOR GLYCOLYSIS AND DC ACTIVATION}

Substantial evidence demonstrates that upon activation immune cells undergo a metabolic reprograming, switching from oxidative phosphorylation (OXPHOS) to aerobic glycolysis, a phenomenon initially observed in cancer cells in 1920s by Warburg (the Warburg effect) (12). In cancer cells, the Warburg effect is induced by growth factor signaling or by mutations in metabolism-related intrinsic pathways [such as loss-of-function mutants of succinate dehydrogenase (SDH) and Fumarate hydratase (FH), and constitutive activation of hypoxia-inducible factor-1 (HIF-1) and (c-Myc)] (13, 14), while $T$ cells undergo the metabolic switch upon $T$ cell receptor (TCR) activation by Ag in the context of proper co-stimulation (15). This change in cellular metabolic pathways provides essential metabolic and bio-energetic resources to support programs of new gene expression and protein synthesis during robust cellular proliferation. $(16,17)$

A recent study from the Pearce group reported that PAMP stimulation of TLR induces a metabolic transition in resting immature DC, characterized by a conversion from mitochondrial $\beta$-oxidation of lipid and OXPHOS (Figure 1A) to aerobic glycolysis (Figure 1B) (18). Unlike in cancer cells and effector T cells, the Warburg effect in DC does not fuel cell division but rather appears to be crucial for DC activation and survival upon TLR stimulation. 


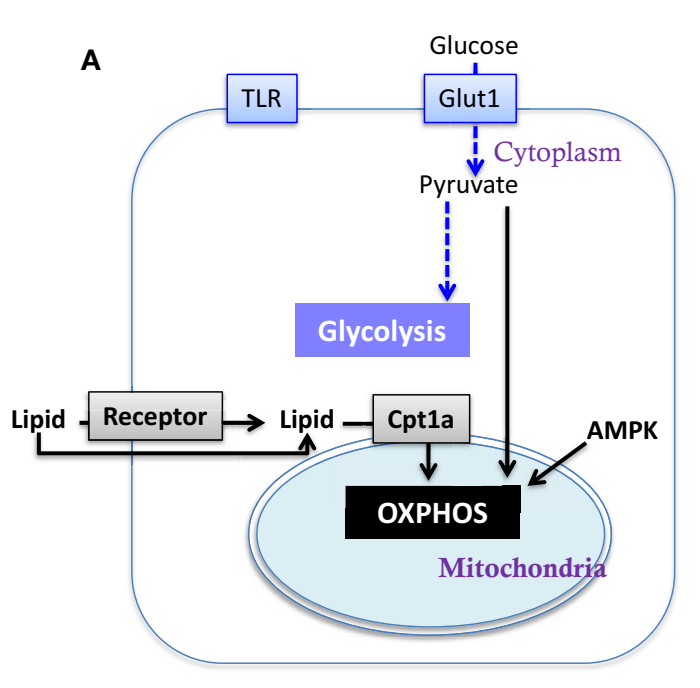

FIGURE 1 | Metabolic regulation of DC activation. Illustrated here are metabolic pathways in resting DC (A) and activated DC (B). Upon TLR stimulation, DC undergo a metabolic switch from OXPHOS (key mediators are linked by black arrows) to glycolysis (key mediators are linked by blue arrows),

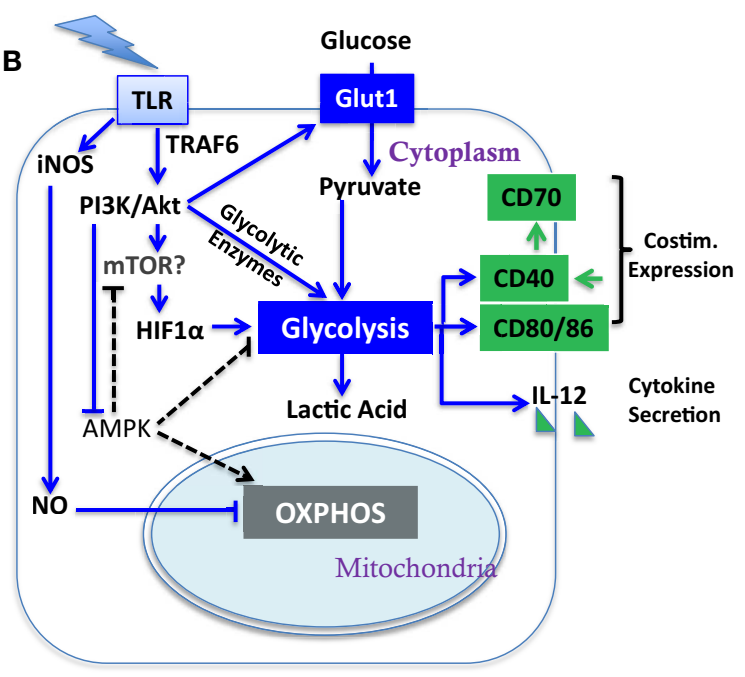

which contributes to their activation (major determinants in green; costim. is short for co-stimulatory molecule). Block arrows mark blockade and dash lines show insignificant process. The impact of mTOR on glycolysis has yet to be fully elucidated in DC (see Section "AKT and mTOR in DC Function" in text).
During the early phase (within $5 \mathrm{~h}$ ) after exposure to TLR agonists, absence of glucose in culture medium led to profound defects in DC activation, including surface expression of CD40 and CD86 and production of IL-12p40 (Figure 1B). Subsequently, DC activated by TLR signals are highly reliant on glucose for survival and become more sensitive to death by nutrient limitation (18). Thus, initiating glycolysis at the time of DC activation is critical for full activation independent from its role in subsequent survival (18). The glycolytic pathway, rather than OXPHOS, may be required due to the need to generate substrates that will be used during DC activation. Alternatively, components of the glycolytic pathway, such as GAPDH, can directly regulate protein translation and may be responsible for regulating the translation of proteins that are critical for DC activation. Further studies will be necessary to elucidate the mechanism by which glycolytic pathway promotes the DC maturation process.

The induction of glycolysis and DC maturation could be influenced by the tumor and the TME at several salient junctures (Figure 2). First, in the context of tumors, it is unclear whether the "find-me, eat-me" signals generated by damage associated molecular patterns (DAMPs)/alarmins, such as nucleotides, uric acid, heat shock proteins (HSP), HMBG1, and calreticulin [which stimulate DC in varied manners including via purinergic receptors (19), CD91 (20), TLR engagement (21, 22), RAGE (23), and TIM-3 (24)], are sufficient to promote glycolysis and DC maturation in the manner achieved with PAMP-mediated stimulation. Second, tumor-derived DC, or DC cultured with tumors, have been shown to be recalcitrant to TLR-mediated induction of CD40, CD86, and IL-12 (25), suggesting that the induction of glycolysis via this pathway in DC may be compromised. The mechanisms that regulate TLR function in mature DC after exposure to tumors have yet to be been elucidated, though inhibition of TLR signaling by MSR1 (see Section "MSR1 and DC Function" below) may contribute.
Third, tumors are highly competitive for glucose; thus the substrate for glycolysis may be unavailable for DC and therefore the TME may not be permissive for the aspects of DC activation that are dependent upon glycolysis.

\section{AKT AND mTOR IN DC FUNCTION}

TLRs activate PI3K in a MyD88-dependent manner (26). Similar to cancer cells $(27,28)$ and effector T cells $(15,29,30)$, PI3K/AKT pathway has been implicated to play a pivotal role in controlling metabolic transition to glycolysis in TLR-stimulated DC (Figure 1B) (18). AKT promotes glycolysis in DC in part by increasing the expression of Glut-1 and likely activates mTOR. In $\mathrm{T}$ cells, AKT signaling promotes glycolysis by inducing the expression of rate-limiting enzymes such as hexokinase and phosphofructokinase (31) and activates mTOR. AKT is not the only driver of metabolic alterations in TLR-stimulated DC, as it is dispensable for the programed down-regulation of palmitate consumption after TLR stimulation. (18). Confounding data exist, however, about the contribution of mTOR (which is normally a downstream target of AKT) to DC immunostimulatory capacity as inhibition of mTOR by rapamycin in murine GM-CSF-driven DC and human myeloid DC prolongs their lifespan, promotes expression of co-stimulatory molecules and cytokines, and enhances DC immunogenicity $(32,33)$. Mouse DC treated with rapamycin were more effective at generating tumor immunity compared to untreated controls. (32). However, in contrast, rapamycin-treated monocyte-derived human DC expressed significantly lower levels of pro-inflammatory cytokines and had reduced capacity to elicit CD8 ${ }^{+} \mathrm{T}$ cell responses (33). Thus, while the TLR-induced activation of DC is dependent upon AKT-mediated induction of glycolysis, the contribution of mTORC1 [which is required to sustain glycolysis and effector functions in T cells (34)] to the glycolytic switch is unresolved, and sustained mTORC1 activation 


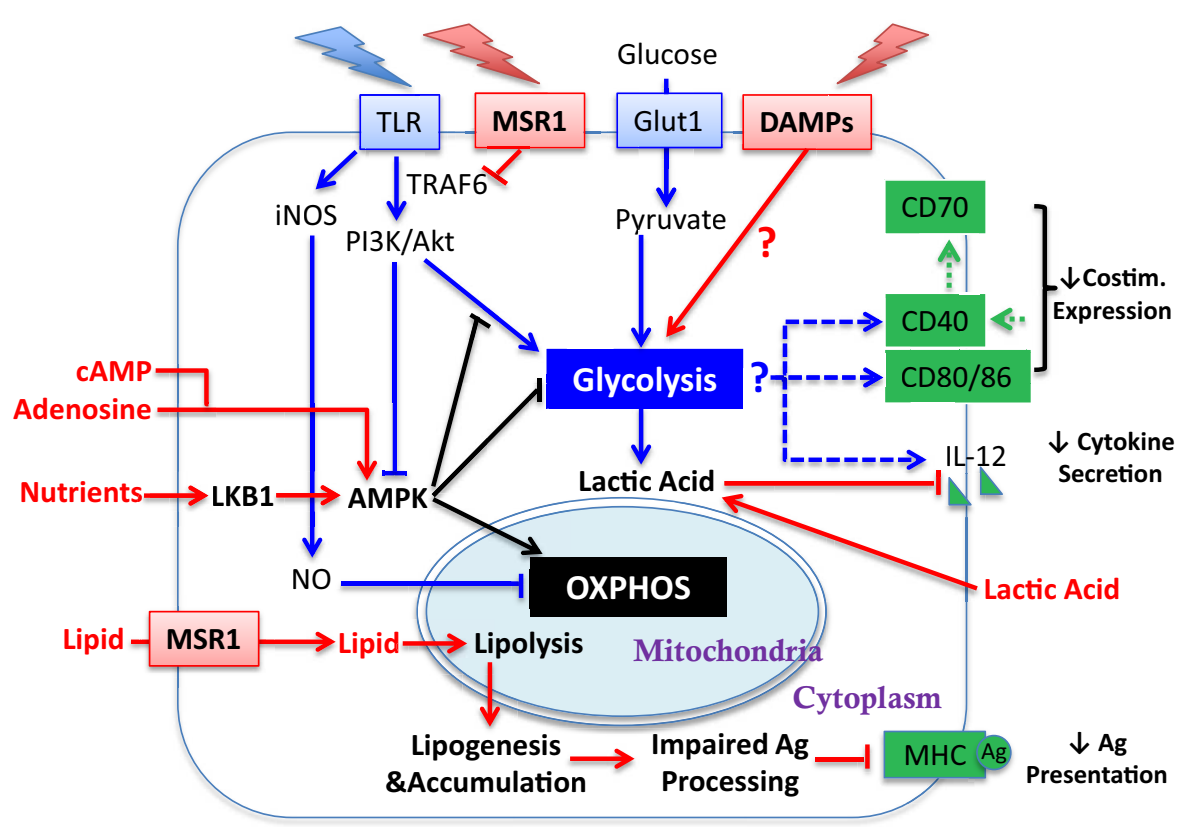

FIGURE 2 | Influence of tumor-derived factors on metabolic regulation of $\mathbf{D C}$ activation. Illustrated here are tumor-derived factors (in red) and their impact/potential impact on metabolic pathways (linked by red arrows) for DC activation. Other symbols are defined the same way as in Figure 1. Lipid accumulation is detrimental to DC activation by impairing Ag processing. How the TME influences glycolytic switch and how that impacts DC activation requires further investigation (see Sections "Requirement for Glycolysis and DC Activation," "Regulation of OXPHOS in DC," and "AMPK Regulation of DC Function"). appears detrimental to the function of DC. Further studies are also needed to dissect the contribution of mTORC2 to any of these processes.

\section{REGULATION OF OXPHOS IN DC}

The underlying mechanisms for the AKT-independent reduction in OXPHOS upon TLR stimulation of DC have been recently studied using real-time metabolic flux analysis (35). The progressive impairment in OXPHOS in TLR-stimulated DC is due to inducible nitric oxide synthase (iNOS)-derived NO (Figure 1B). LPS stimulation induces NOS2 mRNA and iNOS protein expression, and subsequent NO production in DC, presumably via IFN-1 or NF- $\kappa \mathrm{B}$-dependent mechanisms as reported in macrophages (36). The autocrine NO causes mitochondrial impairment and blocks OXPHOS, as reported previously in astrocytes (37) and macrophages (38). The mechanism of OXPHOS inhibition is likely by NO reversibly competing with oxygen to inhibit cytochrome $c$ oxidase, the terminal enzyme of the electron transport chain (39). Thus, the increase in glycolysis in DC after TLR stimulation could be a survival response that serves to maintain cellular ATP levels and to prevent cell death when OXPHOS is blocked and pyruvate accumulates. Most interestingly, although switch to glycolysis has been demonstrated to be a direct consequence of iNOS-mediated OXPHOS blockade and is essential for the survival of iNOS-expressing DC in vivo, a long-term switch to glycolysis was shown to be dispensable for full DC activation. When NO production is inhibited, glycolysis is abrogated and $\beta$ oxidation is maintained in TLR-stimulated DC. Despite this, these DC showed unimpaired if not enhanced activation, as assessed by surface expression of MHC and co-stimulatory molecules, production of inflammatory cytokines, and capacity of DC to stimulate $\mathrm{T}$ cell proliferative responses at $24 \mathrm{~h}$ after TLR stimulation. Therefore, optimal DC function may require only a transient switch to glycolysis; this notion is consistent with the fact that either stimulation of CD40 (see below) or limiting mTOR activity promotes DC function, and raises an alternative hypothesis to explain DC dysfunction in tumors: that rather than being unresponsive to DAMPs, chronic exposure to DAMPS from tumors leads to a state of glycolysis-induced exhaustion or elimination of DC (Figure 2).

\section{AMPK REGULATION OF DC FUNCTION}

The switch to glycolysis by DC is antagonized by adenosine monophosphate-activated protein kinase (AMPK), a master regulator of catabolic metabolism/OXPHOS in eukaryotic cells (Figure 1) $(40,41)$. AMPK can be induced by the nutrient sensor LKB1 (42), and functions in an opposing fashion with PI3K/AKT pathway to regulate TLR-induced metabolism and DC activation (Figure 2): up-regulation of AMPK in DC resulted in decreased LPS-induced IL-12p40 expression and glucose consumption, while suppression of AMPK by shRNAi leads to increased IL-12p40 and CD86 (18). Given the capacity of tumors to compete for glucose, one possible explanation for limited DC activity within the TME is the dominance of AMPK signaling over AKT-driven pathways (Figure 2). Intriguingly, IL-10, an inhibitor of DC activation, has been found to antagonize the TLR-induced hypophosphorylation of AMPK in TLR-stimulated DC, subsequently inhibiting the induction of glycolysis (18). AMPK activity is also strongly 
induced by cAMP (Figure 2). Along with adenosine, cAMP can skew myeloid cell differentiation to a tolerogenic DC format (43, 44) and ablate the function of already differentiated DC (45). It is therefore intriguing to consider whether the negative regulatory activity of cAMP and adenosine in tumor immunity is mediated by abrogating glycolysis. However, the increased immunostimulatory capacity of DC after mTOR blockade or iNOS inhibition is not simply due to a restoration of $\beta$-oxidation. If this were the case, then contrary to these observations, AMPK activation should promote DC immunostimulatory capacity. Further, ligation of CD40, which signals via TRAF-6, has a major influence on DC activation state and viability (46-49), and is a potent promoter of co-stimulatory molecule and IL-12 expression and immunity to cancer. TRAF-6 mediated signaling has been shown to promote fatty acid oxidation in $\mathrm{CD}^{+} \mathrm{T}$ cells $(50)$ via activation of AMPK, raising the question as to how signals generated by CD40 engagement might be integrated into the metabolic programing initiated by TLR signaling. AMPK agonists have been proposed as anti-cancer agents due to their anti-Warburg effect in cancer cells (51), but this approach may be compromised by the detrimental effects of AMPK on DC and $\mathrm{CD}^{+} \mathrm{T}$ cell function. The role of AMPK in regulating glycolysis and $\beta$-oxidation in early and late stages of DC function requires further elucidation before it can be predicted how such an approach would impact on DC function in tumors.

\section{LACTIC ACID REGULATION OF DC FUNCTION}

Additional metabolic regulation of DC has been described. Endogenously produced lactic acid, the end product of glycolysis, accumulates in dense monocyte-derived DC cultures and tumor spheroids (52). Lactic acid concentration after glycolysis, rather than oxygen availability, skews DC differentiation into a tolerogenic orientation, as exemplified by increased production of IL-10 and loss of IL-12 (Figure 2) in response to TLR stimuli (53). This potentially identifies a negative feedback loop in DC function induced by glycolysis within activated DC, and may suggest that the beneficial effect of preventing the switch to glycolysis achieved by inhibiting NO production in vitro (35) could be a secondary consequence of avoiding lactic acid accumulation in DC culture. Interestingly, Ag uptake, MHC class I presentation and costimulatory molecule (CD40 and CD86) expression on DC can be increased by acidosis/extracellular acid (54), via acid-sensing ion channels (ASICs) (55). Thus, acidity and lactate accumulation may be independent variables on DC maturation. Although lactic acid buildup due to excessive DC density is unlikely to be a major consideration in vivo, tumor-generated lactic acid may serve this purpose (Figure 2) $(27,56,57)$. Lactate export by cells is passive, mediated by monocarboxylate transporters (MCTs). A high extracellular lactate concentration in the TME could prevent its export from glycolytic DC, leading to lactate accumulation. It is also worth considering whether lactic acid buildup will differentially affect DC that are resident within tumors compared to those at the tumor edge, and whether lymph node resident DC are vulnerable to lactic acid prior to lymph node invasion by metastatic disease. Further investigations are necessary to establish a comprehensive understanding about how changes in glycolysis and OXPHOS influence activation and/or survival of different TADC subsets, whether DC maturation states are equivalently influenced by metabolic alterations, and whether diverse TLR and other PAMP stimuli have similar impact on DC metabolism.

\section{LIPID UPTAKE AND METABOLISM IN TADC}

While a switch to glycolytic metabolism is generally consistent with immune cell activation, fatty acid metabolism, and lipogenesis are thought to promote quiescence $(17,50)$. Several studies have now begun to illuminate a rather complex role of lipid, and lipid accumulation, in DC function, and how the presence or production of triglycerides (triacylglycerol, TAG) in the context of tumors may influence DC function.

As DC develop and mature, particularly after LPS stimulation, they take on a "lacy" appearance that is composed of an increased presence of fat and glycogen-containing lipid-body droplets (58). Notably, these high lipid DC (HL-DC) express higher levels of scavenger receptors including MARCO/MSR1, which may contribute to their accumulation of lipid (58). Aside from serving as a building block for many facets of DC biology, lipid can contribute to critical aspects of the ability of DC to perform their Ag processing and presentation functions. Cross-presentation of exogenous Ag on MHC class I molecules is highly dependent upon the presence of lipid bodies. Genetic inactivation of genes that regulate lipid-body assembly, or the use of diacylglycerol acyltransferase inhibitors that prevent TAG accumulation, abrogates the MHC class I cross-presenting capability of DC (59). Thus, lipid production and consumption play critical roles in DC biology.

\section{LIPID-MEDIATED INHIBITION OF TADC FUNCTION}

It is therefore of interest that elevated levels of lipid, particularly TAG, were observed first by Herber and colleagues in DC during tumor progression of lymphoma, colon, and breast cancer in preclinical mouse models and cancer patients (60). The observed increased lipid accumulation is primarily a consequence of increased lipid uptake via up-regulated scavenger receptor A (SRA/MSR1/CD204) (Figure 2). Remarkably, considering the data from Bourgneres et al. (59), the major functional defect in HL-DC was a reduced capacity of DC to cross-present Ag (60). Normalization of lipid levels by a pharmacological inhibitor of acetyl-CoA carboxylase-1 (ACC-1), an enzyme that plays a critical role in lipogenesis, restored functional activity of lipid-laden DC, and enabled them to become more potent when used in a cancer vaccine (60). There are several notable aspects of this study that are worthy of further consideration. First, as mentioned above, lipid in DC by itself is not necessarily a marker of dysfunction. Indeed, a recent study examined the immunogenic qualities of liver-derived DC containing high and low amounts of lipid. HL-DC were considerably more immunogenic than their low lipid counterparts across multiple measurements (61). Further, Hwang and colleagues have demonstrated that saturated fatty acids can activate TLR4, leading to the up-regulation of MHC and costimulatory molecules. In contrast, polyunsaturated fats such as DHA, counteract the ability of saturated fats to induce DC maturation (62). Thus, rather than the amount of lipid within a DC being detrimental to function, the process by which lipid is acquired, or synthesized, or the type of lipid (saturated versus unsaturated) may be influential on DC function. 


\section{MSR1 AND DC FUNCTION}

MSR1 has been shown to act both as a lipid receptor and as an innate pattern recognition receptor (PRR) that regulates inflammatory responses. As the first receptor identified for modified lipoproteins, the role of MSR1 has been well explored in pathogenesis of vascular disease particularly atherosclerosis (63). Besides modified self macromolecules, a wide range of PAMPs have been identified as MSR1 ligands, including bacterial surface components (e.g., LPS) and nucleic acids (e.g., CpG DNA and double-strand RNA), apoptotic cells, and endogenous danger molecules (64). Notably, the first report about negative effects of MSR1 in DC activation and function during adaptive immunity was presented by Yi and colleagues (65), in which they demonstrated that MSR1 suppresses the ability of TLR4 stimulation to license DC to prime naive CD8 T cells, drive their expansion, and promote their cytotoxic functionality both in vitro and in vivo (65). In agreement with data from Herber et al. they showed that lack of MSR1 in hematopoietic cells promoted tumor-protective immunity in a B16-OVA mouse melanoma model. In this model, MSR1 suppressed TLR4-induced activation of the transcription factor NF- $\kappa \mathrm{B}$ by directly inhibiting ubiquitination of TRAF-6 (Figure 2) (66). However, the restriction of NF- $\mathrm{B}$ activity by MSR1 can be independent of its ligand-binding domain, implying a novel signaling-regulatory role of MSR1 that can be uncoupled from its conventional role in endocytosis, including lipid uptake. Accordingly, one can speculate that up-regulation of MSR1 can contribute to DC dysfunction in cancer by skewing at least two pathways: (1) accumulation of lipids (2) suppression of TLR signaling. With respect to the second point, inhibition of TLR signaling may alter the balance between lipolysis and lipogenesis in favor of lipid accumulation. The therapeutic relevance of MSR1 and lipid uptake is reinforced by studies showing that direct targeting of MSR1 promotes tumor immunity $(67,68)$. Further, recent studies by Lerret et al. showed that the ability of total body irradiation (TBI), in combination with adoptive transfer of tumor-specific $\mathrm{CD}^{+}$ $\mathrm{T}$ cells, to control established breast tumors may be achieved by promoting activation and function in tumor-resident DC via down-regulating MSR1 and inhibition of lipid uptake (69, 70). However, the tumor-derived factors that up-regulate MSR1 are poorly characterized, and it is yet to be definitively shown that lipid is an immunoregulatory ligand for MSR1on DC.

\section{MSR1-INDEPENDENT EFFECTS OF LIPID ON TADC}

Although MSR1 engagement could account for poor DC function, additional influences of lipid on DC cannot be ruled out. Inhibition of ACC-1 resulted in normalization of lipid levels in TADC and was sufficient to restore functional activity in lipid-laden DC without changing expression of MHC and costimulatory molecules (60). This indicates that at least some accumulation of lipid in DC is due to de novo lipogenesis (Figure 2). Further, either the detrimental effects of lipid accumulation can be independent of MSR1 (as ACC-1 inhibition refunctionalizes TADC), or pathways released by ACC-1 inhibition can overcome MSR1-mediated inhibition. Evidence for the latter concept has been provide by Rehman et al. in a study demonstrating that ACC-1 inhibition enhances Ag capture (rather than Ag processing) by human DC (71). Confounding our understanding is that ACC-1 regulates the production of malonyl CoA, which in turn inhibits the activity of Carnitine palmitoyltransferase Ia (Cptla) (Figure 1A). Cptla strongly suppresses glycolysis via the Randle cycle, and knockdown of Cptla has been shown to strongly promote glycolysis in T cells (72). Thus, it is unclear why the inhibition of ACC-1, which should reduce glycolysis, enhances DC function unless (1) the lipogenesis program activates pathways that are significantly deleterious to DC function; (2) sustained glycolysis is indeed detrimental to DC function (discussed above); or (3) the presence of lipid is the detrimental factor, by influencing the availability of pyruvate for glycolysis rather than OXPHOS (73). Pointedly, it is uncertain why the accumulation of lipid might be detrimental to DC function at the level of Ag processing and presentation, especially given the importance of lipid bodies in this process. However, it has been shown that ceramides, which due to their hydrophobicity could accumulate in fat droplets, abrogate the ability of DC to uptake and present $\mathrm{Ag}$ (74) and also promote tumor-induced DC apoptosis (75).

\section{IN VITRO VERITAS?}

While the emerging picture of how alterations in DC metabolism can influence the function of DC, several words of caution should be written. One noticeable aspect of the majority of the studies cited in this review is that analysis of the contribution of metabolic alterations to DC function has generally been performed on DC generated from bone marrow or PBMC. This is necessitated by the rarity of DC in tissues, and the low sensitivity of the assays that are currently available to characterize metabolic activity. Thus, it is possible to posit that some metabolism-associated alterations described in these studies could be dependent upon the culture conditions that generate or sustain DC, and extrapolation to in vivo DC, particular to intratumoral DC, is not yet merited. DC, particular those of murine origin, generated via culture exist in a semi-activated functional state (our unpublished data) that may lead to different metabolic choices, and be influenced by different stimuli, compared to truly immature DC. This point is particularly salient when we consider some of the reported discrepancies on the impact of limiting or promoting glycolysis by modulating mTOR activity. Further, much work has yet to be done in defining whether metabolic alterations actually promote discrete functions of DC, or whether metabolic switching is a response to alterations in the nutrients in the immediate environment of the DC (tissue; lymphatics; lymph nodes, for example). However, the capacity for TLR to induce metabolic changes in DC in the consistent nutrient environment provided by in vitro culture, suggests that metabolic changes are not entirely due to alterations in the available nutrients, but rather these metabolic changes directly impact/regulate the activation and survival of DC. The single-cell analytical luxuries provided by flow cytometry have yet to be translated to metabolism studies, limiting our ability to make direct assessment of in vivo DC metabolic changes. Unfortunately, until radiotracer incorporation, extracellular flux assays, and mass spectrometry can be applied to 1000 s of cells, rather than 100,000 s, we will be dependent upon the use of fluorochrome-labeled substrates such as the glucose-derivative 2-NBDG to guide our impression of the metabolic pathways being used by DC derived from different in vivo environments. 


\section{SUMMARY}

The metabolic and biochemical regulation of DC activation, function, and survival are just the beginning to be elucidated. Further understanding of this process will likely improve the quality and efficacy of DC expanded ex vivo for cancer vaccines [note the varied influences of cytokines on vaccine efficacy $(9,76)]$, as cytokines are known to impact metabolism. Further, metabolic re-invigoration of DC may provide an avenue for enhancing DC function in the TME or in tumor-draining lymph nodes, allowing for increased Ag processing and presentation after the induction of tumor damage, or in association with inhibition of checkpoint blockade molecules. Finally, approaches that promote the availability of glucose, or limit lipid uptake, in the TME might well increase the ability of TADC to activate and contribute to the adaptive immune responses elicited against tumors.

\section{ACKNOWLEDGMENTS}

Research in Timothy N. J. Bullock's laboratory is funded by the Melanoma Research Alliance and 1R01CA166458-01A1. We would like to thank Dr. Kyle Hoehn and Lelisa Gemta for critiquing the manuscript and providing important comments.

\section{REFERENCES}

1. Waithman J, Allan RS, Kosaka H, Azukizawa H, Shortman K, Lutz MB, et al. Skin-derived dendritic cells can mediate deletional tolerance of class I-restricted self-reactive T cells. J Immunol (2007) 179:4535-41.

2. Appleman LJ, Boussiotis VA. T cell anergy and costimulation. Immunol Rev (2003) 192:161-80. doi:10.1034/j.1600-065X.2003.00009.x

3. Randolph GJ, Angeli V, Swartz MA. Dendritic-cell trafficking to lymph nodes through lymphatic vessels. Nat Rev Immunol (2005) 5:617-28. doi:10.1038/ nri1670

4. Alvarez D, Vollmann EH, von Andrian UH. Mechanisms and consequences of dendritic cell migration. Immunity (2008) 29:325-42. doi:10.1016/j.immuni. 2008.08.006

5. Bullock TN, Yagita H. Induction of CD70 on dendritic cells through CD40 or TLR stimulation contributes to the development of CD8+ T cell responses in the absence of CD4+ T cells. J Immunol (2005) 174:710-7.

6. Van Deusen KE, Rajapakse R, Bullock TN. CD70 expression by dendritic cells plays a critical role in the immunogenicity of CD40-independent, CD4+ T celldependent, licensed CD8+ T cell responses. J Leukoc Biol (2010) 87:477-85. doi:10.1189/jlb.0809535

7. Feau S, Garcia Z, Arens R, Yagita H, Borst J, Schoenberger SP. The CD4(+) T-cell help signal is transmitted from APC to CD8(+) T-cells via CD27-CD70 interactions. Nat Commun (2012) 3:948. doi:10.1038/ncomms 1948

8. Hammer GE, Ma A. Molecular control of steady-state dendritic cell maturation and immune homeostasis. Annu Rev Immunol (2013) 31:743-91. doi:10.1146/annurev-immunol-020711-074929

9. Palucka K, Banchereau J. Dendritic-cell-based therapeutic cancer vaccines. Immunity (2013) 39:38-48. doi:10.1016/j.immuni.2013.07.004

10. Treilleux I, Blay JY, Bendriss-Vermare N, Ray-Coquard I, Bachelot T, Guastalla JP, et al. Dendritic cell infiltration and prognosis of early stage breast cancer. Clin Cancer Res (2004) 10:7466-74. doi:10.1158/1078-0432.CCR-04-0684

11. Perrot I, Blanchard D, Freymond N, Isaac S, Guibert B, Pacheco Y, et al. Dendritic cells infiltrating human non-small cell lung cancer are blocked at immature stage. J Immunol (2007) 178:2763-9.

12. Warburg O, Posener K, Negelein E. Ueber den Stoffwechsel der Tumoren. Biochem Zeitschr (1924) 152:319-44.

13. Frezza C, Pollard PJ, Gottlieb E. Inborn and acquired metabolic defects in cancer. J Mol Med (Berl) (2011) 89:213-20. doi:10.1007/s00109-011-0728-4

14. Porporato PE, Dhup S, Dadhich RK, Copetti T, Sonveaux P. Anticancer targets in the glycolytic metabolism of tumors: a comprehensive review. Front Pharmacol (2011) 2:49. doi:10.3389/fphar.2011.00049

15. Frauwirth KA, Riley JL, Harris MH, Parry RV, Rathmell JC, Plas DR, et al. The CD28 signaling pathway regulates glucose metabolism. Immunity (2002) 16:769-77. doi:10.1016/S1074-7613(02)00323-0
16. Palsson-McDermott EM, O'Neill LA. The Warburg effect then and now: from cancer to inflammatory diseases. Bioessays (2013) 35:965-73. doi:10.1002/bies. 201300084

17. Pearce EL, Pearce EJ. Metabolic pathways in immune cell activation and quiescence. Immunity (2013) 38:633-43. doi:10.1016/j.immuni.2013.04.005

18. Krawczyk CM, Holowka T, Sun J, Blagih J, Amiel E, DeBerardinis RJ, et al. Tolllike receptor-induced changes in glycolytic metabolism regulate dendritic cell activation. Blood (2010) 115:4742-9. doi:10.1182/blood-2009-10-249540

19. Elliott MR, Chekeni FB, Trampont PC, Lazarowski ER, Kadl A, Walk SF, et al. Nucleotides released by apoptotic cells act as a find-me signal to promote phagocytic clearance. Nature (2009) 461:282-6. doi:10.1038/nature08296

20. Binder RJ, Han DK, Srivastava PK. CD91: a receptor for heat shock protein gp 96. Nat Immunol (2000) 1:151-5. doi:10.1038/77835

21. Vabulas RM, Wagner H, Schild H. Heat shock proteins as ligands of toll-like receptors. Curr Top Microbiol Immunol (2002) 270:169-84.

22. Park JS, Svetkauskaite D, He Q, Kim JY, Strassheim D, Ishizaka A, et al. Involvement of toll-like receptors 2 and 4 in cellular activation by high mobility group box 1 protein. J Biol Chem (2004) 279:7370-7. doi:10.1074/jbc.M306793200

23. Yang D, Chen Q, Yang H, Tracey KJ, Bustin M, Oppenheim JJ. High mobility group box-1 protein induces the migration and activation of human dendritic cells and acts as an alarmin. J Leukoc Biol (2007) 81:59-66. doi:10.1189/jlb. 0306180

24. Chiba S, Baghdadi M, Akiba H, Yoshiyama H, Kinoshita I, Dosaka-Akita H, et al. Tumor-infiltrating DCs suppress nucleic acid-mediated innate immune responses through interactions between the receptor TIM-3 and the alarmin HMGB1. Nat Immunol (2012) 13:832-42. doi:10.1038/ni.2376

25. Idoyaga J, Moreno J, Bonifaz L. Tumor cells prevent mouse dendritic cell maturation induced by TLR ligands. Cancer Immunol Immunother (2007) 56:1237-50. doi:10.1007/s00262-006-0275-y

26. Gelman AE, LaRosa DF, Zhang J, Walsh PT, Choi Y, Sunyer JO, et al. The adaptor molecule MyD88 activates PI-3 kinase signaling in CD4+ T cells and enables CpG oligodeoxynucleotide-mediated costimulation. Immunity (2006) 25:783-93. doi:10.1016/j.immuni.2006.08.023

27. Levine AJ, Puzio-Kuter AM. The control of the metabolic switch in cancers by oncogenes and tumor suppressor genes. Science (2010) 330:1340-4. doi:10.1126/science.1193494

28. Ran C, Liu H, Hitoshi Y, Israel MA. Proliferation-independent control of tumor glycolysis by PDGFR-mediated AKT activation. Cancer Res (2013) 73:1831-43. doi:10.1158/0008-5472.CAN-12-2460

29. Engelman JA, Luo J, Cantley LC. The evolution of phosphatidylinositol 3kinases as regulators of growth and metabolism. Nat Rev Genet (2006) 7:606-19. doi:10.1038/nrg1879

30. Jones RG, Thompson CB. Revving the engine: signal transduction fuels $\mathrm{T}$ cell activation. Immunity (2007) 27:173-8. doi:10.1016/j.immuni.2007.07.008

31. Frauwirth KA, Thompson CB. Regulation of $\mathrm{T}$ lymphocyte metabolism. J Immunol (2004) 172:4661-5.

32. Amiel E, Everts B, Freitas TC, King IL, Curtis JD, Pearce EL, et al. Inhibition of mechanistic target of rapamycin promotes dendritic cell activation and enhances therapeutic autologous vaccination in mice. J Immunol (2012) 189:2151-8. doi:10.4049/jimmunol.1103741

33. Haidinger M, Poglitsch M, Geyeregger R, Kasturi S, Zeyda M, Zlabinger GJ, et al. A versatile role of mammalian target of rapamycin in human dendritic cell function and differentiation. J Immunol (2010) 185:3919-31. doi:10.4049/ jimmunol.1000296

34. Finlay DK, Rosenzweig E, Sinclair LV, Feijoo-Carnero C, Hukelmann JL, Rolf $J$, et al. PDK1 regulation of mTOR and hypoxia-inducible factor 1 integrate metabolism and migration of CD8+ T cells. J Exp Med (2012) 209:2441-53. doi:10.1084/jem.20112607

35. Everts B, Amiel E, van der Windt GJ, Freitas TC, Chott R, Yarasheski KE, et al. Commitment to glycolysis sustains survival of NO-producing inflammatory dendritic cells. Blood (2012) 120:1422-31. doi:10.1182/blood-2012-03-419747

36. Lewis RS, Kolesnik TB, Kuang Z, D’Cruz AA, Blewitt ME, Masters SL, et al. TLR regulation of SPSB1 controls inducible nitric oxide synthase induction. J Immunol (2011) 187:3798-805. doi:10.4049/jimmunol.1002993

37. Bolanos JP, Peuchen S, Heales SJ, Land JM, Clark JB. Nitric oxide-mediated inhibition of the mitochondrial respiratory chain in cultured astrocytes. JNeurochem (1994) 63:910-6. doi:10.1046/j.1471-4159.1994.63030910.x

38. Garedew A, Moncada S. Retraction. Mitochondrial dysfunction and HIFlalpha stabilization in inflammation. J Cell Sci (2012) 125:3254. doi:10.1242/jcs.115949 
39. Cleeter MW, Cooper JM, Darley-Usmar VM, Moncada S, Schapira AH. Reversible inhibition of cytochrome c oxidase, the terminal enzyme of the mitochondrial respiratory chain, by nitric oxide. Implications for neurodegenerative diseases. FEBS Lett (1994) 345:50-4. doi:10.1016/0014-5793(94)00424-2

40. Hardie DG. Roles of the AMP-activated/SNF1 protein kinase family in the response to cellular stress. Biochem Soc Symp (1999) 64:13-27.

41. Hardie DG, Ross FA, Hawley SA. AMPK: a nutrient and energy sensor that maintains energy homeostasis. Nat Rev Mol Cell Biol (2012) 13:251-62. doi:10.1038/nrm3311

42. Tamas P, Macintyre A, Finlay D, Clarke R, Feijoo-Carnero C, Ashworth A, et al. LKB1 is essential for the proliferation of T-cell progenitors and mature peripheral T cells. Eur J Immunol (2010) 40:242-53. doi:10.1002/eji.200939677

43. Novitskiy SV, Ryzhov S, Zaynagetdinov R, Goldstein AE, Huang Y, Tikhomirov $\mathrm{OY}$, et al. Adenosine receptors in regulation of dendritic cell differentiation and function. Blood (2008) 112:1822-31. doi:10.1182/blood-2008-02-136325

44. Challier J, Bruniquel D, Sewell AK, Laugel B. Adenosine and cAMP signalling skew human dendritic cell differentiation towards a tolerogenic phenotype with defective CD8(+) T-cell priming capacity. Immunology (2013) 138:402-10. doi:10.1111/imm.12053

45. Panther E, Durk T, Ferrari D, Di Virgilio F, Grimm M, Sorichter S, et al. AMP affects intracellular Ca2+ signaling, migration, cytokine secretion and $\mathrm{T}$ cell priming capacity of dendritic cells. PLoS One (2012) 7:e37560. doi:10.1371/ journal.pone.0037560

46. Miga AJ, Masters SR, Durell BG, Gonzalez M, Jenkins MK, Maliszewski C, et al. Dendritic cell longevity and T cell persistence is controlled by CD154-CD40 interactions. Eur J Immunol (2001) 31:959-65. doi:10.1002/1521-4141(200103) 31:3<959::AID-IMMU959>3.0.CO;2-A

47. Mackey MF, Wang Z, Eichelberg K, Germain RN. Distinct contributions of different CD40 TRAF binding sites to CD154-induced dendritic cell maturation and IL-12 secretion. Eur J Immunol (2003) 33:779-89. doi:10.1002/eji.200323729

48. Kobayashi T, Walsh PT, Walsh MC, Speirs KM, Chiffoleau E, King CG, et al. TRAF6 is a critical factor for dendritic cell maturation and development. Immunity (2003) 19:353-63. doi:10.1016/S1074-7613(03)00230-9

49. Elgueta R, Benson MJ, de Vries VC, Wasiuk A, Guo Y, Noelle RJ. Molecular mechanism and function of CD40/CD40L engagement in the immune system. Immunol Rev (2009) 229:152-72. doi:10.1111/j.1600-065X.2009.00782.x

50. Pearce EL, Walsh MC, Cejas PJ, Harms GM, Shen H, Wang LS, et al. Enhancing CD8 T-cell memory by modulating fatty acid metabolism. Nature (2009) 460:103-7. doi:10.1038/nature08097

51. Faubert B, Boily G, Izreig S, Griss T, Samborska B, Dong Z, et al. AMPK is a negative regulator of the Warburg effect and suppresses tumor growth in vivo. Cell Metab (2013) 17:113-24. doi:10.1016/j.cmet.2012.12.001

52. Gottfried E, Kunz-Schughart LA, Ebner S, Mueller-Klieser W, Hoves S, Andreesen R, et al. Tumor-derived lactic acid modulates dendritic cell activation and antigen expression. Blood (2006) 107:2013-21. doi:10.1182/blood-200505- 1795

53. Nasi A, Fekete T, Krishnamurthy A, Snowden S, Rajnavolgyi E, Catrina AI, et al. Dendritic cell reprogramming by endogenously produced lactic acid. J Immunol (2013) 191:3090-9. doi:10.4049/jimmunol.1300772

54. Vermeulen M, Giordano M, Trevani AS, Sedlik C, Gamberale R, FernandezCalotti $\mathrm{P}$, et al. Acidosis improves uptake of antigens and MHC class I-restricted presentation by dendritic cells. J Immunol (2004) 172:3196-204.

55. Tong J, Wu WN, Kong X, Wu PF, Tian L, Du W, et al. Acid-sensing ion channels contribute to the effect of acidosis on the function of dendritic cells. J Immunol (2011) 186:3686-92. doi:10.4049/jimmunol.1001346

56. Hirschhaeuser F, Sattler UG, Mueller-Klieser W. Lactate: a metabolic key player in cancer. Cancer Res (2011) 71:6921-5. doi:10.1158/0008-5472.CAN-11-1457

57. Dhup S, Dadhich RK, Porporato PE, Sonveaux P. Multiple biological activities of lactic acid in cancer: influences on tumor growth, angiogenesis and metastasis. Curr Pharm Des (2012) 18:1319-30. doi:10.2174/138161212799504902

58. Maroof A, English NR, Bedford PA, Gabrilovich DI, Knight SC. Developing dendritic cells become 'lacy' cells packed with fat and glycogen. Immunology (2005) 115:473-83. doi:10.1111/j.1365-2567.2005.02181.x

59. Bougneres L, Helft J, Tiwari S, Vargas P, Chang BH, Chan L, et al. A role for lipid bodies in the cross-presentation of phagocytosed antigens by MHC class I in dendritic cells. Immunity (2009) 31:232-44. doi:10.1016/j.immuni. 2009.06.022

60. Herber DL, Cao W, Nefedova Y, Novitskiy SV, Nagaraj S, Tyurin VA, et al. Lipid accumulation and dendritic cell dysfunction in cancer. Nat Med (2010) 16:880-6. doi:10.1038/nm.2172
61. Ibrahim J, Nguyen AH, Rehman A, Ochi A, Jamal M, Graffeo CS, et al. Dendritic cell populations with different concentrations of lipid regulate tolerance and immunity in mouse and human liver. Gastroenterology (2012) 143:1061-72. doi:10.1053/j.gastro.2012.06.003

62. Weatherill AR, Lee JY, Zhao L, Lemay DG, Youn HS, Hwang DH. Saturated and polyunsaturated fatty acids reciprocally modulate dendritic cell functions mediated through TLR4. J Immunol (2005) 174:5390-7.

63. Suzuki H, Kurihara Y, Takeya M, Kamada N, Kataoka M, Jishage K, et al. A role for macrophage scavenger receptors in atherosclerosis and susceptibility to infection. Nature (1997) 386:292-6. doi:10.1038/386292a0

64. Areschoug T, Gordon S. Scavenger receptors: role in innate immunity and microbial pathogenesis. Cell Microbiol (2009) 11:1160-9. doi:10.1111/j.1462-5822. 2009.01326.x

65. Yi H, Yu X, Gao P, Wang Y, Baek SH, Chen X, et al. Pattern recognition scavenger receptor SRA/CD204 down-regulates Toll-like receptor 4 signaling-dependent CD8 T-cell activation. Blood (2009) 113:5819-28. doi:10.1182/blood-2008-11190033

66. Yu X, Yi H, Guo C, Zuo D, Wang Y, Kim HL, et al. Pattern recognition scavenger receptor CD204 attenuates Toll-like receptor 4-induced NF-kappaB activation by directly inhibiting ubiquitination of tumor necrosis factor (TNF) receptorassociated factor 6. J Biol Chem (2011) 286:18795-806. doi:10.1074/jbc.M111. 224345

67. Yi H, Guo C, Yu X, Gao P, Qian J, Zuo D, et al. Targeting the immunoregulator SRA/CD204 potentiates specific dendritic cell vaccine-induced T-cell response and antitumor immunity. Cancer Res (2011) 71:6611-20. doi:10.1158/00085472.CAN-11-1801

68. Guo C, Yi H, Yu X, Zuo D, Qian J, Yang G, et al. In situ vaccination with CD204 gene-silenced dendritic cell, not unmodified dendritic cell, enhances radiation therapy of prostate cancer. Mol Cancer Ther (2012) 11:2331-41. doi:10.1158/1535-7163.MCT-12-0164

69. Lerret NM, Rogozinska M, Jaramillo A, Marzo AL. Adoptive transfer of mammaglobin-A epitope specific CD8 T cells combined with a single low dose of total body irradiation eradicates breast tumors. PLoS One (2012) 7:e41240. doi:10.1371/journal.pone.0041240

70. Lerret NM, Marzo AL. Adoptive T-cell transfer combined with a single low dose of total body irradiation eradicates breast tumors. Oncoimmunology (2013) 2:e22731. doi:10.4161/onci.22731

71. Rehman A, Hemmert KC, Ochi A, Jamal M, Henning JR, Barilla R, et al. Role of fatty-acid synthesis in dendritic cell generation and function. J Immunol (2013) 190:4640-9. doi:10.4049/jimmunol.1202312

72. van der Windt GJ, Everts B, Chang CH, Curtis JD, Freitas TC, Amiel E, et al. Mitochondrial respiratory capacity is a critical regulator of CD8+ T cell memory development. Immunity (2012) 36:68-78. doi:10.1016/j.immuni.2011.12.007

73. Sabbah HH, Stanley WC. Partial fatty acid oxidation inhibitors: a potentially new class of drugs for heart failure. Eur J Heart Fail (2002) 4:3-6. doi:10.1016/S1388-9842(01)00183-0

74. Sallusto F, Nicolo C, De Maria R, Corinti S, Testi R. Ceramide inhibits antigen uptake and presentation by dendritic cells. J Exp Med (1996) 184:2411-6. doi:10.1084/jem.184.6.2411

75. Kanto T, Kalinski P, Hunter OC, Lotze MT, Amoscato AA. Ceramide mediates tumor-induced dendritic cell apoptosis. J Immunol (2001) 167:3773-84.

76. Palucka K, Banchereau J. Cancer immunotherapy via dendritic cells. Nat Rev Cancer (2012) 12:265-77. doi:10.1038/nrc3258

Conflict of Interest Statement: The authors declare that the research was conducted in the absence of any commercial or financial relationships that could be construed as a potential conflict of interest.

Received: 19 November 2013; accepted: 16 January 2014; published online: 30 January 2014.

Citation: Dong H and Bullock TNJ (2014) Metabolic influences that regulate dendritic cell function in tumors. Front. Immunol. 5:24. doi: 10.3389/fimmu.2014.00024

This article was submitted to Tumor Immunity, a section of the journal Frontiers in Immunology.

Copyright () 2014 Dong and Bullock. This is an open-access article distributed under the terms of the Creative Commons Attribution License (CC BY). The use, distribution or reproduction in other forums is permitted, provided the original author (s) or licensor are credited and that the original publication in this journal is cited, in accordance with accepted academic practice. No use, distribution or reproduction is permitted which does not comply with these terms. 